\title{
A Family of Stiffly Stable Second Derivative Block Methods for Initial Value Problems in Ordinary Differential Equations
}

\author{
Samuel A. Ajayi ${ }^{1}$, Kingsley O. Muka ${ }^{2}$ and Oluwasegun M. Ibrahim ${ }^{3, *}$ \\ ${ }^{1}$ Advanced Research Laboratory, Department of Mathematics, University of Benin, Benin City, Nigeria; \\ e-mail: ajayiadegbuyi@gmail.com \\ ${ }^{2}$ Advanced Research Laboratory, Department of Mathematics, University of Benin, Benin City, Nigeria; \\ e-mail: kingsley.muka@uniben.edu \\ ${ }^{3}$ African Institute for Mathematical Sciences, Kigali, Rwanda; e-mail: oluwasegun.micheal@aims.ac.rw \\ * Corresponding Author
}

\begin{abstract}
In this paper, we present a family of stiffly stable second derivative block methods (SDBMs) suitable for solving first-order stiff ordinary differential equations (ODEs). The methods proposed herein are consistent and zero stable, hence, they are convergent. Furthermore, we investigate the local truncation error and the region of absolute stability of the SDBMs. A flowchart, describing this procedure is illustrated. Some of the developed schemes are shown to be A-stable and L-stable, while some are found to be $A(\alpha)$-stable. The numerical results show that our SDBMs are stiffly stable and give better approximations than the existing methods in the literature.
\end{abstract}

\section{Introduction}

Ordinary differential equations are very important when modeling physical systems in engineering and sciences. Examples of such systems are circuit theory, chemical kinetics, control theory, celestial mechanics and biology. The solution equations of these systems give insight into how the systems evolve and what the effects of changes in the

Received: February 21, 2019; Accepted: March 22, 2019

2010 Mathematics Subject Classification: 65L06, 65L10.

Keywords and phrases: second derivative, A-stability, L-stability, block method, ordinary differential equations.

Copyright (C) 2019 Samuel A. Ajayi et al. This is an open access article distributed under the Creative Commons Attribution License, which permits unrestricted use, distribution, and reproduction in any medium, provided the original work is properly cited. 
systems are. Consequently, the ODEs emanating from the real-life phenomena are highly non-linear and cannot be solved analytically. Hence, we need appropriate numerical methods to solve these problems. The problem of deriving more advanced and efficient methods for stiff problems has received a great deal of attention since the emergence of the famous theorem of Dahlquist [7]. As in Dahlquist [7], a potentially good numerical method for the solution of stiff systems of ODEs must have good accuracy and reasonable wide region of absolute stability. As a matter of necessity, we will investigate the accuracy and the stability of the method to be presented in this paper through numerical experiment and stability analysis.

The backward differentiation formula (BDF) was introduced by Curtiss and Hirschfelder [6] and further development was made by Cash [4]. This was achieved by proposing a class of extended BDF of high order ODEs. Similarly, Chartier [5] and Fatunla [9] have independently improved on the BDF by deriving A-stable methods suitable for the integration of first-order initial value problems (IVPs). The search for higher order A-stable multistep methods is carried out in the two main directions: (1) use higher derivatives of the solutions, (2) throw in additional stages, off-step points, superfuture points. This leads into the large field general linear methods Hairer and Wanner [14]. However, the super-future points could be likened to the super-implicit points presented in Ibrahim and Ikhile [16], Ibrahim and Ikhile [17] respectively for the case of special second-order linear multistep methods. Several authors have considered the second and higher derivatives methods because of the existence of A-stable higher multiderivative schemes, see for example, Enright [10], Ismail and Ibrahim [18], Kumleng and Sirisena [20]. Also, the combination of one-step procedures and the Runge-Kutta procedures was introduced by Gear [11]. In like manner, some numerical analysts have considered the development of this form of methods. Examples are the works presented in Butcher [3], Gupta [12], Mehdizadeh and Molayi [23], Ngwane and Jator [26]. Lambert [19] opined that Milne in 1953 introduced block method for the sole aim of obtaining starting values for predictor-corrector algorithms. Furthermore, Rosser [28] generalized the idea of Milne into an algorithm, and nowadays, several authors have employed Rosser's approach to developing efficient numerical schemes. These authors include Ajie et al. [1], Akinfenwa et al. [2], Majid et al. [21] and Muka and Ikhile [24].

In this work, we consider the block method for the numerical integration of stiff ODEs. We are spur by the fact that block methods are capable of obtaining numerical solutions at several points simultaneously as well as effectively formulated scheme 
capable of solving stiff IVPs in ODEs (stiffly stable). This paper is organized as follows: In Section 2, the formulation of second derivative block methods are considered as well as the pictorial representation of the algorithm. In Section 3, some basic properties of the block methods were investigated and analyzed. The numerical solutions and comparison have been drawn for some methods in Section 4 while the conclusion of the work is stated in Section 5.

We seek the numerical solution of the stiff IVP

$$
y^{\prime}=f(x, y), \quad y(a)=y_{0}, \quad a \leq x \leq b
$$

where $a$ and $b$ are a finite interval, i.e., $I=[a, b], y: I \rightarrow R^{m}$ and $f: I \rightarrow R^{m} \rightarrow R^{m}$ is continuous and differentiable.

In Zabidi et al. [30], the two-point block method of the form

$$
A^{(0)} Y_{m}=A^{(1)} Y_{m-1}+h\left(B^{(0)} F_{m}+B^{(1)} Y_{m-1}\right)
$$

is proposed, where

$$
A^{(0)}=\left[\begin{array}{cc}
1 & 0 \\
-1 & 1
\end{array}\right], \quad A^{(1)}=\left[\begin{array}{ll}
0 & 1 \\
0 & 0
\end{array}\right], \quad B^{(0)}=\left[\begin{array}{cc}
\frac{8}{12} & -\frac{1}{12} \\
\frac{8}{12} & \frac{5}{12}
\end{array}\right], \quad B^{(1)}=\left[\begin{array}{cc}
0 & \frac{5}{12} \\
0 & -\frac{1}{12}
\end{array}\right]
$$

and

$$
Y_{m}=\left[\begin{array}{c}
y_{n+1} \\
y_{n+2}
\end{array}\right], \quad Y_{m-1}=\left[\begin{array}{c}
y_{n-1} \\
y_{n}
\end{array}\right], \quad F_{m}=\left[\begin{array}{c}
f_{n+1} \\
f_{n+2}
\end{array}\right], \quad F_{m-1}=\left[\begin{array}{c}
f_{n-1} \\
f_{n}
\end{array}\right]
$$

\section{Development of the Second Derivative Block Method}

Second derivative block method (SDBM) with high order is derived herein, the continuous form of (2) is proposed and extended to second derivative block formulation. In the spirit of Zabidi et al. [30], the $k$-point, $r$ block SDBM under consideration is of the form

$$
A^{(0)} Y_{m}=A^{(1)} Y_{m-1}+h\left[B^{(0)} F\left(Y_{m}\right)+B^{(1)} F\left(Y_{m-1}\right)\right]+h^{2}\left[C^{(0)} G\left(Y_{m}\right)\right],
$$

where $k$ is the number of points and $r$ is the number of blocks, $h$ is the mesh size, the matrix $G\left(Y_{m}\right)=F^{\prime}\left(Y_{m}\right)$ and the matrix $C^{(0)}$ are strictly diagonal matrices with 
dimension $k \times k$. The general structure of the coefficient matrices for method (3) are as given below

$$
\begin{gathered}
A^{(0)}=\left(\begin{array}{ccccc}
1 & 0 & 0 & \cdots & 0 \\
-1 & 1 & 0 & \cdots & \vdots \\
0 & -1 & 1 & \cdots & \vdots \\
\vdots & 0 & -1 & 1 & 0 \\
0 & 0 & \cdots & -1 & 1
\end{array}\right), \quad A^{(1)}=\left(\begin{array}{ccccc}
0 & 0 & \cdots & 0 & 1 \\
0 & 0 & 0 & 0 & 0 \\
0 & 0 & 0 & \vdots & \vdots \\
\vdots & 0 & 0 & \vdots & \vdots \\
0 & 0 & \cdots & 0 & 0
\end{array}\right), \\
B^{(0)}=\left(\begin{array}{cccc}
b_{11} & b_{12} & \cdots & b_{1 k} \\
b_{21} & b_{22} & \cdots & b_{2 k} \\
\vdots & \vdots & \vdots & \vdots \\
b_{k 1} & b_{k 2} & \cdots & b_{k k}
\end{array}\right), \quad B^{(1)}=\left(\begin{array}{cccc}
0 & 0 & \cdots & b_{1 k} \\
0 & 0 & \cdots & b_{2 k} \\
\vdots & \vdots & \vdots & \vdots \\
0 & 0 & \cdots & b_{k k}
\end{array}\right), \\
C^{(0)}=\left(\begin{array}{cccc}
1 & 0 & \cdots & 0 \\
0 & 1 & \cdots & 0 \\
\vdots & \vdots & \vdots & \vdots \\
0 & 0 & \cdots & 1
\end{array}\right) .
\end{gathered}
$$

The vectors $Y_{m}, Y_{m-1}, F_{m}, F_{m-1}, G_{m}$ and $G_{m-1}$ are defined by

$$
\begin{gathered}
Y_{m}=\left(\begin{array}{c}
y_{n+1} \\
y_{n+2} \\
\vdots \\
y_{n+k}
\end{array}\right) ; \quad Y_{m-1}=\left(\begin{array}{c}
y_{n-k+1} \\
\vdots \\
y_{n-1} \\
y_{n}
\end{array}\right) ; \quad F_{m}=\left(\begin{array}{c}
f_{n+1} \\
f_{n+2} \\
\vdots \\
f_{n+k}
\end{array}\right) ; \\
F_{m-1}=\left(\begin{array}{c}
f_{n-k+1} \\
\vdots \\
f_{n-1} \\
f_{n}
\end{array}\right) ; \quad G_{m}=\left(\begin{array}{c}
g_{n+1} \\
g_{n+2} \\
\vdots \\
g_{n+k}
\end{array}\right) ; \quad G_{m-1}=\left(\begin{array}{c}
g_{n-1} \\
\vdots \\
g_{n-1} \\
g_{n}
\end{array}\right),
\end{gathered}
$$

where $\left(b_{11}, \ldots, b_{k 1}\right)^{T},\left(b_{12}, \ldots, b_{k 2}\right)^{T}$ and $\left(b_{1 k}, \ldots, b_{k k}\right)^{T}$ are the coefficients to be determined. The entries in matrices $A^{(0)}$ in $A^{(1)}$ are fixed to ensure desirable stability criteria. Hence, we present the flowchart of the formulation of the SDBM for first-order IVPs in ODEs. 
Development of the SDBM is based on the application of the scalar test problems (STPs)

$$
y^{\prime}=\lambda y, \quad y^{\prime \prime}=\lambda^{2} y .
$$

\section{The presentation of the general form}

of the SDBM under considearation is given by

$$
\Upsilon: A^{(0)} Y_{m}=A^{(1)} Y_{m-1}+h\left[B^{(0)} F\left(Y_{m}\right)+B^{(1)} F\left(Y_{m-1}\right)\right]+h^{2}\left[C^{(0)} G\left(Y_{m}\right)\right],
$$

\section{where:}

1. $\left\{x_{j}\right\}_{j=0}^{r} \in[a, b]$ and $[a, b]$ is the integration region,

2. the stepsize of the integration $h=\left|x_{j+1}-x_{j}\right|, j=0(1) r$.

$$
\Upsilon \rightarrow \begin{cases}B^{r} \neq 0 & \text { implicit }, \\ B^{r}=0 & \text { explicit. }\end{cases}
$$

\section{On the application of the general STP}

on the general form of the SDBM $\Upsilon$ yields the stability polinomial that determines the region of absolute stability.

Figure 1. Flowchart algorithm for the development of the SDBM.

\section{Construction of the Second Derivative Block Method (SDBM)}

In this section, we construct up to seven points second derivative block method using Mathematica Software, Borwein and Skerritt [32]. 


\subsection{Two-point second derivative block method (SDBM)}

To derive the two-point SDBM of the form (3), we set

$$
\begin{gathered}
A^{(0)}=\left(\begin{array}{cc}
1 & 0 \\
-1 & 1
\end{array}\right), \quad A^{(1)}=\left(\begin{array}{ll}
0 & 1 \\
0 & 0
\end{array}\right), \quad B^{(0)}=\left(\begin{array}{ll}
b_{11} & b_{12} \\
b_{21} & b_{22}
\end{array}\right), \\
B^{(1)}=\left(\begin{array}{ll}
0 & b_{12} \\
0 & b_{22}
\end{array}\right), \quad C^{(0)}=\left(\begin{array}{cc}
c_{11} & 0 \\
0 & c_{22}
\end{array}\right),
\end{gathered}
$$

where the corresponding vectors

$$
\begin{gathered}
Y_{m}=\left[\begin{array}{c}
y_{n+1} \\
y_{n+2}
\end{array}\right] ; \quad Y_{m-1}=\left[\begin{array}{c}
y_{n-1} \\
y_{n}
\end{array}\right] ; \\
F_{m}=\left[\begin{array}{c}
f_{n+1} \\
f_{n+2}
\end{array}\right] ; \quad F_{m-1}=\left[\begin{array}{c}
f_{n-1} \\
f_{n}
\end{array}\right] ; \quad G_{m}=\left[\begin{array}{c}
g_{n+1} \\
g_{n+2}
\end{array}\right] .
\end{gathered}
$$

The algebraic coefficients obtained for the two-point SDBM yields

$$
\begin{gathered}
A^{(0)}=\left(\begin{array}{cc}
1 & 0 \\
-1 & 1
\end{array}\right), \quad A^{(1)}=\left(\begin{array}{ll}
0 & 1 \\
0 & 0
\end{array}\right), \quad B^{(0)}=\left(\begin{array}{cc}
\frac{16}{24} & \frac{1}{24} \\
\frac{20}{48} & \frac{29}{48}
\end{array}\right), \\
B^{(1)}=\left(\begin{array}{cc}
0 & \frac{7}{24} \\
0 & -\frac{1}{48}
\end{array}\right), \quad C^{(0)}=\left(\begin{array}{cc}
-\frac{6}{24} & 0 \\
0 & -\frac{6}{48}
\end{array}\right) .
\end{gathered}
$$

Hence, the block form (4) can be written in the linear form

$$
\begin{gathered}
y_{n+1}=y_{n}+\frac{h}{24}\left(7 f_{n}+16 f_{n+1}+f_{n+2}\right)+\frac{h^{2}}{24}\left(-6 g_{n+1}\right), \\
y_{n+2}=y_{n+1}+\frac{h}{48}\left(f_{n}+20 f_{n+1}+29 f_{n+2}\right)+\frac{h^{2}}{48}\left(-6 g_{n+2}\right) .
\end{gathered}
$$

In similar fashion, we derive for $k=3, \ldots, 7$. 


\subsection{Three-point second derivative block method}

The three-point SDBM for $k=3$ is given by

$$
\begin{gathered}
A^{(0)}=\left(\begin{array}{ccc}
1 & 0 & 0 \\
-1 & 1 & 0 \\
0 & -1 & 1
\end{array}\right), \quad A^{(1)}=\left(\begin{array}{lll}
0 & 0 & 1 \\
0 & 0 & 0 \\
0 & 0 & 0
\end{array}\right), \\
B^{(0)}=\left(\begin{array}{ccc}
\frac{228}{360} & \frac{39}{360} & -\frac{4}{360} \\
\frac{129}{360} & \frac{228}{360} & \frac{7}{360} \\
-\frac{54}{1080} & \frac{513}{1080} & \frac{614}{1080}
\end{array}\right), \quad B^{(1)}=\left(\begin{array}{ccc}
0 & 0 & \frac{97}{360} \\
0 & 0 & -\frac{4}{360} \\
0 & 0 & \frac{7}{1080}
\end{array}\right), \\
C^{(0)}=\left(\begin{array}{ccc}
-\frac{114}{360} & 0 & 0 \\
0 & -\frac{66}{360} & 0 \\
0 & 0 & -\frac{114}{1080}
\end{array}\right) .
\end{gathered}
$$

\subsection{Four-point second derivative block method}

The four-point SDBM for $k=4$ is given by

$$
\begin{gathered}
A^{(0)}=\left(\begin{array}{cccc}
1 & 0 & 0 & 0 \\
-1 & 1 & 0 & 0 \\
0 & -1 & 1 & 0 \\
0 & 0 & -1 & 1
\end{array}\right), \quad A^{(1)}=\left(\begin{array}{llll}
0 & 0 & 0 & 1 \\
0 & 0 & 0 & 0 \\
0 & 0 & 0 & 0 \\
0 & 0 & 0 & 0
\end{array}\right), \\
B^{(0)}=\left(\begin{array}{cccc}
\frac{842}{1440} & \frac{282}{1440} & -\frac{58}{1440} & \frac{7}{1440} \\
\frac{944}{2880} & \frac{1824}{2880} & \frac{144}{2880} & -\frac{11}{2880} \\
-\frac{114}{4320} & \frac{1746}{4320} & \frac{2626}{4320} & \frac{51}{4320} \\
\frac{690464}{17280} & -\frac{1554156}{17280} & \frac{2079264}{17280} & -\frac{1068851}{17280}
\end{array}\right), \quad B^{(1)}=\left(\begin{array}{cccc}
0 & 0 & 0 & -\frac{540}{1440} \\
0 & 0 & 0 & -\frac{21}{2880} \\
0 & 0 & 0 & \frac{11}{4320} \\
0 & 0 & 0 & -\frac{129441}{17280}
\end{array}\right),
\end{gathered}
$$




$$
C^{(0)}=\left(\begin{array}{cccc}
-\frac{540}{1440} & 0 & 0 & 0 \\
0 & -\frac{660}{2880} & 0 & 0 \\
0 & 0 & -\frac{660}{4320} & 0 \\
0 & 0 & 0 & \frac{515940}{17280}
\end{array}\right) \text {. }
$$

\subsection{Five-point second derivative block method}

The five-point SDBM for $k=5$ is given by

$$
\begin{aligned}
& A^{(0)}=\left(\begin{array}{ccccc}
1 & 0 & 0 & 0 & 0 \\
-1 & 1 & 0 & 0 & 0 \\
0 & -1 & 1 & 0 & 0 \\
0 & 0 & -1 & 1 & 0 \\
0 & 0 & 0 & -1 & 1
\end{array}\right), \quad A^{(1)}=\left(\begin{array}{ccccc}
0 & 0 & 0 & 0 & 1 \\
0 & 0 & 0 & 0 & 0 \\
0 & 0 & 0 & 0 & 0 \\
0 & 0 & 0 & 0 & 0 \\
0 & 0 & 0 & 0 & 0
\end{array}\right), \\
& B^{(0)}=\left(\begin{array}{ccccc}
\frac{63773}{120960} & \frac{36528}{120960} & -\frac{11292}{120960} & \frac{2728}{120960} & -\frac{321}{120960} \\
-\frac{6208}{20160} & \frac{12501}{20160} & \frac{1808}{20160} & -\frac{277}{20160} & \frac{27}{20160} \\
-\frac{347}{20160} & \frac{7408}{20160} & \frac{12501}{20160} & \frac{608}{20160} & -\frac{37}{20160} \\
\frac{1048}{120960} & -\frac{5412}{120960} & \frac{53328}{120960} & \frac{71123}{120960} & \frac{984}{120960} \\
-\frac{7935}{604800} & \frac{29840}{604800} & -\frac{76260}{604800} & \frac{340440}{604800} & \frac{317731}{604800}
\end{array}\right), \\
& B^{(1)}=\left(\begin{array}{ccccc}
0 & 0 & 0 & 0 & \frac{29544}{120960} \\
0 & 0 & 0 & 0 & -\frac{107}{20160} \\
0 & 0 & 0 & 0 & \frac{27}{20160} \\
0 & 0 & 0 & 0 & -\frac{111}{120960} \\
0 & 0 & 0 & 0 & \frac{984}{604800}
\end{array}\right), \quad C^{(0)}=\left(\begin{array}{ccccc}
0 & 0 & 0 & 0 & -\frac{51780}{120960} \\
0 & 0 & 0 & 0 & \frac{5420}{20160} \\
0 & 0 & 0 & 0 & -\frac{3820}{20160} \\
0 & 0 & 0 & 0 & -\frac{16260}{120960} \\
0 & 0 & 0 & 0 & -\frac{51780}{604800}
\end{array}\right) .
\end{aligned}
$$




\subsection{Six-point second derivative block method}

The six-point SDBM for $k=6$ is given by

$$
\begin{aligned}
& A^{(0)}=\left(\begin{array}{cccccc}
1 & 0 & 0 & 0 & 0 & 0 \\
-1 & 1 & 0 & 0 & 0 & 0 \\
0 & -1 & 1 & 0 & 0 & 0 \\
0 & 0 & -1 & 1 & 0 & 0 \\
0 & 0 & 0 & -1 & 1 & 0 \\
0 & 0 & 0 & 0 & -1 & 1
\end{array}\right), \quad A^{(1)}=\left(\begin{array}{cccccc}
0 & 0 & 0 & 0 & 0 & 1 \\
0 & 0 & 0 & 0 & 0 & 0 \\
0 & 0 & 0 & 0 & 0 & 0 \\
0 & 0 & 0 & 0 & 0 & 0 \\
0 & 0 & 0 & 0 & 0 & 0 \\
0 & 0 & 0 & 0 & 0 & 0
\end{array}\right), \\
& B^{(0)}=\left(\begin{array}{cccccc}
\frac{112223}{241920} & \frac{102906}{241920} & -\frac{42484}{241920} & \frac{15406}{241920} & -\frac{3627}{241920} & \frac{398}{241920} \\
\frac{146387}{406560} & \frac{2801749}{4181760} & -\frac{4157}{101640} & \frac{11551}{406560} & -\frac{152347}{18295200} & \frac{1123}{1084160} \\
-\frac{503}{40320} & \frac{13861}{40320} & \frac{586}{945} & \frac{2171}{40320} & -\frac{53}{8064} & \frac{191}{362880} \\
\frac{271}{60480} & -\frac{781}{26880} & \frac{12067}{30240} & \frac{293347}{483840} & \frac{139}{6720} & -\frac{253}{241920} \\
-\frac{109}{26880} & \frac{257}{13440} & -\frac{3971}{60480} & \frac{6347}{13440} & \frac{76861}{134400} & \frac{731}{120960} \\
\frac{179}{20160} & -\frac{5771}{161280} & \frac{8131}{90720} & -\frac{13823}{80640} & \frac{12079}{20160} & \frac{247021}{483840}
\end{array}\right), \\
& B^{(1)}=\left(\begin{array}{cccccc}
0 & 0 & 0 & 0 & 0 & \frac{57098}{241920} \\
0 & 0 & 0 & 0 & 0 & -\frac{41779}{4065600} \\
0 & 0 & 0 & 0 & 0 & \frac{289}{362880} \\
0 & 0 & 0 & 0 & 0 & -\frac{191}{483840} \\
0 & 0 & 0 & 0 & 0 & \frac{253}{604800} \\
0 & 0 & 0 & 0 & 0 & -\frac{731}{725760}
\end{array}\right)
\end{aligned}
$$




$C^{(0)}=\left(\begin{array}{cccccc}\frac{115500}{241920} & 0 & 0 & 0 & 0 & 0 \\ 0 & -\frac{10843}{69696} & 0 & 0 & 0 & 0 \\ 0 & 0 & -\frac{191}{864} & 0 & 0 & 0 \\ 0 & 0 & 0 & -\frac{191}{1152} & 0 & 0 \\ 0 & 0 & 0 & 0 & -\frac{39}{320} & 0 \\ 0 & 0 & 0 & 0 & 0 & -\frac{275}{3456}\end{array}\right)$.

\subsection{The coefficients of seven-point second derivative block method}

The seven-point SDBM for $k=7$ is given by

$$
\begin{gathered}
A^{(0)}=\left(\begin{array}{ccccccc}
1 & 0 & 0 & 0 & 0 & 0 & 0 \\
-1 & 1 & 0 & 0 & 0 & 0 & 0 \\
0 & -1 & 1 & 0 & 0 & 0 & 0 \\
0 & 0 & -1 & 1 & 0 & 0 & 0 \\
0 & 0 & 0 & -1 & 1 & 0 & 0 \\
0 & 0 & 0 & 0 & -1 & 1 & 0 \\
0 & 0 & 0 & 0 & 0 & -1 & 0
\end{array}\right), \quad A^{(1)}=\left(\begin{array}{ccccccc}
0 & 0 & 0 & 0 & 0 & 0 & 0 \\
0 & 0 & 0 & 0 & 0 & 0 & 0 \\
0 & 0 & 0 & 0 & 0 & 0 & 0 \\
0 & 0 & 0 & 0 & 0 & 0 & 0 \\
0 & 0 & 0 & 0 & 0 & 0 & 0
\end{array}\right), \\
B^{(0)}=\left(\begin{array}{cccccccc}
\frac{449527}{1134000} & \frac{341699}{604800} & -\frac{105943}{362880} & \frac{153761}{1088640} & -\frac{943}{18900} & \frac{99359}{9072000} & -\frac{6031}{5443200} \\
\frac{512669}{1814400} & \frac{2600231}{4536000} & \frac{13985}{72576} & -\frac{21509}{362880} & \frac{31111}{1814400} & -\frac{3047}{907200} & \frac{409}{1296000} \\
-\frac{17483}{1814400} & \frac{197611}{604800} & \frac{13903}{22680} & \frac{29843}{362880} & -\frac{9127}{604800} & \frac{13169}{5443200} & -\frac{23}{113400} \\
\frac{14639}{5443200} & -\frac{12697}{604800} & \frac{135053}{362880} & \frac{13903}{22680} & \frac{22261}{604800} & -\frac{6773}{1814400} & \frac{199}{777600} \\
-\frac{1577}{907200} & \frac{17881}{1814400} & -\frac{15419}{362880} & \frac{30911}{72576} & \frac{2692841}{4536000} & \frac{27779}{1814400} & -\frac{1201}{1814400} \\
\frac{20609}{9072000} & -\frac{52}{4725} & \frac{37631}{1088640} & -\frac{32233}{362880} & \frac{302429}{604800} & \frac{633277}{1134000} & \frac{8563}{1814400} \\
-\frac{35453}{5443200} & \frac{86791}{302400} & -\frac{2797}{36288} & \frac{157513}{1088640} & -\frac{133643}{604800} & \frac{1147051}{1814400} & \frac{1758023}{3528000}
\end{array}\right),
\end{gathered}
$$




$$
B^{(1)}=\left(\begin{array}{ccccccc}
0 & 0 & 0 & 0 & 0 & 0 & \frac{416173}{1814400} \\
0 & 0 & 0 & 0 & 0 & 0 & \frac{6031}{1814400} \\
0 & 0 & 0 & 0 & 0 & 0 & \frac{409}{777600} \\
0 & 0 & 0 & 0 & 0 & 0 & -\frac{23}{113400} \\
0 & 0 & 0 & 0 & 0 & 0 & \frac{199}{1296000} \\
0 & 0 & 0 & 0 & 0 & 0 & -\frac{1201}{5443200} \\
0 & 0 & 0 & 0 & 0 & 0 & \frac{8563}{12700800}
\end{array}\right)
$$

$$
C^{(0)}=\left(\begin{array}{ccccccc}
-\frac{33953}{64800} & 0 & 0 & 0 & 0 & 0 & 0 \\
0 & -\frac{7297}{21600} & 0 & 0 & 0 & 0 & 0 \\
0 & 0 & -\frac{3233}{12960} & 0 & 0 & 0 & 0 \\
0 & 0 & 0 & -\frac{2497}{12960} & 0 & 0 & 0 \\
0 & 0 & 0 & 0 & -\frac{3233}{21600} & 0 & 0 \\
0 & 0 & 0 & 0 & 0 & -\frac{7297}{64800} & 0 \\
0 & 0 & 0 & 0 & 0 & 0 & -\frac{33953}{453600}
\end{array}\right) .
$$

\section{Stability Analysis of Methods}

Theorem 1 (Chartier [5]). Let $e=[1,1,1, \ldots, 1]^{T}, c=[1,2,3, \ldots, k]^{T}$ and the order condition for k-point r-block method (3) be given as

$$
\begin{aligned}
& C_{0}=e-\sum_{j=1}^{k} A_{j} e \\
& C_{1}=c-\sum_{j=1}^{k} A_{j}(c-\mu j e)-\sum_{j=0}^{k} B_{j} e
\end{aligned}
$$




$$
\begin{aligned}
& C_{2}=c^{2}-\sum_{j=1}^{k} A_{j}(c-\mu j e)^{2}-2 \sum_{j=0}^{k} B_{j}(c-\mu j e) \\
& \vdots \\
& \vdots \\
& C_{i}=c^{i}-\sum_{j=1}^{k} A_{j}(c-\mu j e)^{i}-i \sum_{j=0}^{k} B_{j}(c-\mu j e)^{i-1} ; \quad i=3,4,5, \ldots
\end{aligned}
$$

The powers of vectors in (7) are component-wise. The SDBM (3) are of order p, if $C_{j}=0, \forall j=0,1, \ldots, p$. It can be verified that the coefficients of (3) for k-point SDBM is order $p=k+2$.

Theorem 2. The application of the scalar test equations

$$
y^{\prime}=\lambda y, \quad y^{\prime \prime}=\lambda^{2} y, \quad \operatorname{Re}(\lambda)>0
$$

on the method (3) yields the stability polynomial

$$
Y_{m}=M(z) Y_{m-1}, \quad z=\lambda h .
$$

The matrix $M(z)$ is called the amplification matrix and it is given by

$$
M(z)=\left(A^{(0)}-z B^{(0)}-z^{2} C^{(0)}\right)^{-1}\left(A^{(1)}+z B^{(1)}\right) .
$$

The polynomial $\pi(w, z)$ associated with (3) is given by

$$
\pi(w, z)=\operatorname{Det}\left[I_{k} w-M(z)\right] .
$$

As in Fatunla [9], we state the preliminary definitions.

Definition 1. The block method (3) is zero stable provided the roots $R_{j}, j=1, \ldots, k$ of the first characteristics polynomial $\rho(R)$ specified by

$$
\rho(r)=\operatorname{det}\left[\sum_{i=0}^{k} A^{(i)} R^{k-1}\right]=0, \quad A^{(0)}=I
$$

satisfied $\left|R_{j}\right| \leq 1, j=1, \ldots, k$, and for those roots with $\left|R_{j}\right|=1$, the multiplicity does not exceed 1 . 
Definition 2. The block method (3) is consistent if it has order at least one.

Definition 3. The block method (3) is convergent if and only if it is consistent and zero stable.

Definition 4. The block method (3) is said to be A-stable if the region of absolute stability (RAS) includes the entire left half of the complex $z$ plane. That is

$$
R A S \supseteq\{z: \operatorname{Re}(z)<0\} .
$$

Definition 5. The block method (3) is said to be $A(\alpha)$-stable with $\alpha \in\left[0, \frac{\pi}{2}\right]$, if the wedge

$$
S_{\alpha}=\{z:|\arg (-z)|<a, z \neq 0\}
$$

is contained in its $R A S$.

Definition 6. The block method (3) is L-stable if it is A-stable and if in addition

$$
\lim _{z \rightarrow \infty} R(z)=0
$$

The RAS of the SDBM (3) is given in Figure 2 while the summary of the error constant and the order of the methods are presented in Table 1.

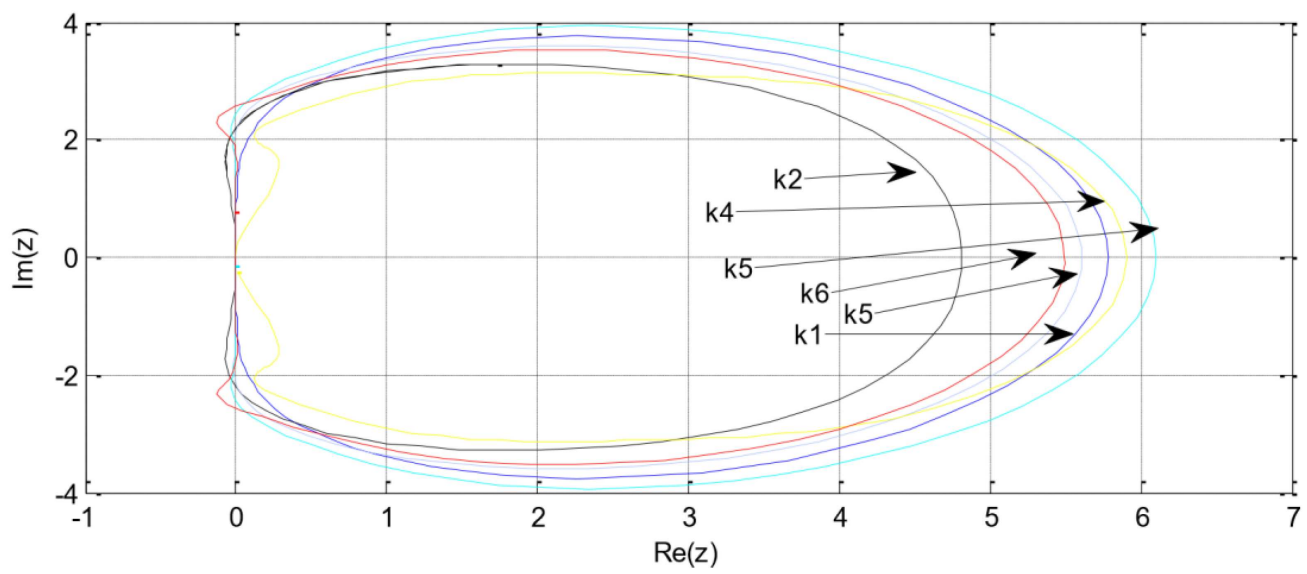

Figure 2. The RAS of the SDBM $(k=1(1) 6)$. 
Table 1. The summary of the error constant and the order of the SDBM (3).

\begin{tabular}{|c|c|c|}
\hline$k$ & Error Constant $C_{p+2}$ & Order $p$ \\
\hline 2 & $\left(-\frac{1}{180}, \frac{7}{1440}\right)^{T}$ & 4 \\
\hline 3 & $\left(\frac{7}{2400},-\frac{11}{7200}, \frac{17}{7200}\right)^{T}$ & 5 \\
\hline 4 & $\left(-\frac{107}{60480}, \frac{1}{512}, \frac{1487}{60480},-\frac{422633}{30240}\right)^{T}$ & 6 \\
\hline 5 & $\left(\frac{199}{169344},-\frac{289}{846720}, \frac{191}{846720},-\frac{253}{846720}, \frac{431177231}{192036096000}\right)^{T}$ & 7 \\
\hline 6 & $\begin{array}{l}\left(-\frac{6031}{7257600},-\frac{793291}{878169600},-\frac{23}{226800},\right. \\
\left.\frac{199}{2073600},-\frac{1201}{7257600}, \frac{8563}{14515200}\right)^{T}\end{array}$ & 8 \\
\hline 7 & $\begin{array}{c}\left(\frac{5741}{9331200},-\frac{2687}{21772800}, \frac{3391}{65318400},-\frac{2497}{65318400},\right. \\
\left.\frac{41}{870912},-\frac{6533}{65318400}, \frac{27719}{65318400}\right)^{T}\end{array}$ & 9 \\
\hline
\end{tabular}

\section{Numerical Experiments}

In this section, the $k$-point SDBM (3) is tested on some given problems and the numerical results are compared to exact solution, existing solutions in the literature, and the solution generated by MATLAB Software ODE 15 Otto and Denier [33].

Problem 1. Consider the initial value problem Zabidi et al. [30]:

$$
y^{\prime}=-10 x y, \quad y(0)=1 \quad 0 \leq x \leq 10 .
$$

The exact solution: $y(x)=e^{-5 x^{2}}$. 
Table 4.1. Error results for problem 1.

\begin{tabular}{|c|c|c|c|}
\hline$h$ & Musa et al. [31] & Zabidi et al. [30] & Two-point SDBM \\
\hline $10^{-1}$ & - & $9.37 \times 10^{(-4)}$ & $6.21 \times 10^{(-5)}$ \\
\hline $10^{-2}$ & $1.24 \times 10^{(-2)}$ & $1.25 \times 10^{(-7)}$ & $7.28 \times 10^{(-8)}$ \\
\hline $10^{-3}$ & $7.36 \times 10^{(-4)}$ & $4.58 \times 10^{(-8)}$ & $7.28 \times 10^{(-11)}$ \\
\hline $10^{-4}$ & $7.07 \times 10^{(-5)}$ & $4.55 \times 10^{(-8)}$ & $0.00 \times 10^{(0)}$ \\
\hline
\end{tabular}

Problem 2. Consider the initial value problem, Zabidi et al. [30]:

$$
y^{\prime}=-100\left(y-x^{3}\right)+3 x^{2}, \quad y(0)=0 \quad 0 \leq x \leq 10 .
$$

The eigenvalue is $\lambda=-100$. Exact solution: $y(x)=x^{3}$.

Table 4.2. Error results for problem 2.

\begin{tabular}{|c|c|c|}
\hline$h$ & Zabidi et al. [30] & Two-point SDBM \\
\hline $10^{-1}$ & $2.41 \times 10^{(-7)}$ & $2.16 \times 10^{(-7)}$ \\
\hline $10^{-2}$ & $4.00 \times 10^{(-7)}$ & $1.24 \times 10^{(-8)}$ \\
\hline $10^{-3}$ & $6.07 \times 10^{(-8)}$ & $1.47 \times 10^{(-11)}$ \\
\hline $10^{-4}$ & $9.21 \times 10^{(-8)}$ & $4.03 \times 10^{(-13)}$ \\
\hline
\end{tabular}

Problem 3. Consider the nonlinear stiff system, Hairer et al. [13]:

$$
\begin{gathered}
y_{1}^{\prime}=y_{2} \\
y_{2}^{\prime}=-y_{2}+\mu y_{2}\left(1-y_{1}^{2}\right): \quad y_{1}(0)=2, \quad y_{2}(0)=0 .
\end{gathered}
$$

The Van der pol equation describes the oscillations in an electrical circuit. The SDBM of order four of our method with step size $h=0.001$ was compared with that of ODE 15, and the resulting phase diagram is given in Figure 3. 


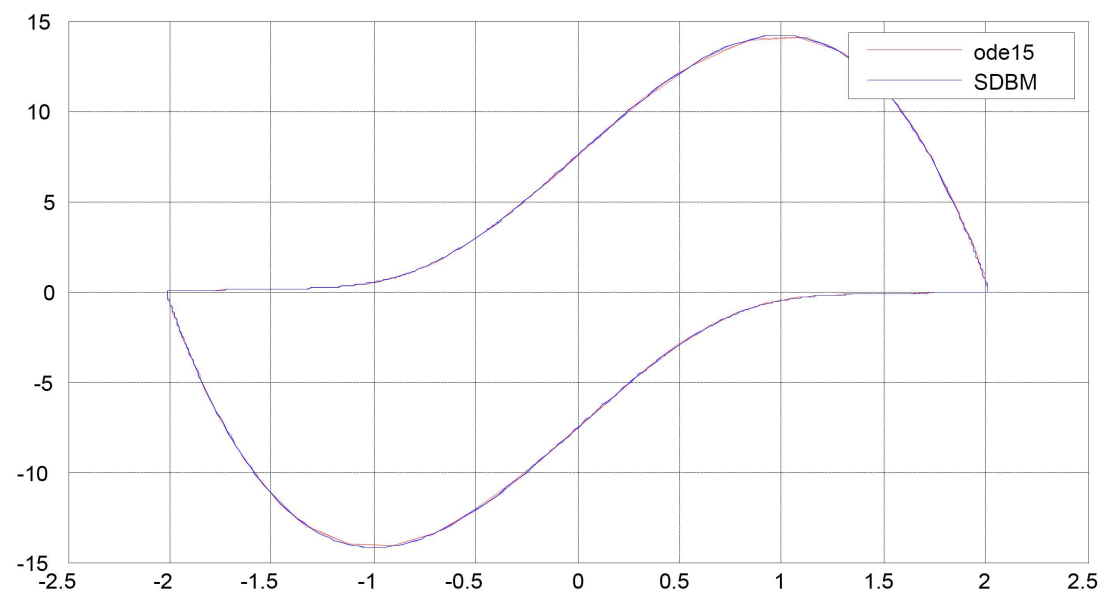

Figure 3. Numerical solution for problem 3 in phase diagram.

Problem 4. Consider the nonlinear problem, Akinfenwa et al. [2]

$$
y^{\prime}=-\frac{y_{3}}{2}, \quad y(0)=1
$$

for $t \in[0, t]$ with the exact solution: $y=\frac{1}{\sqrt{t+1}}$.

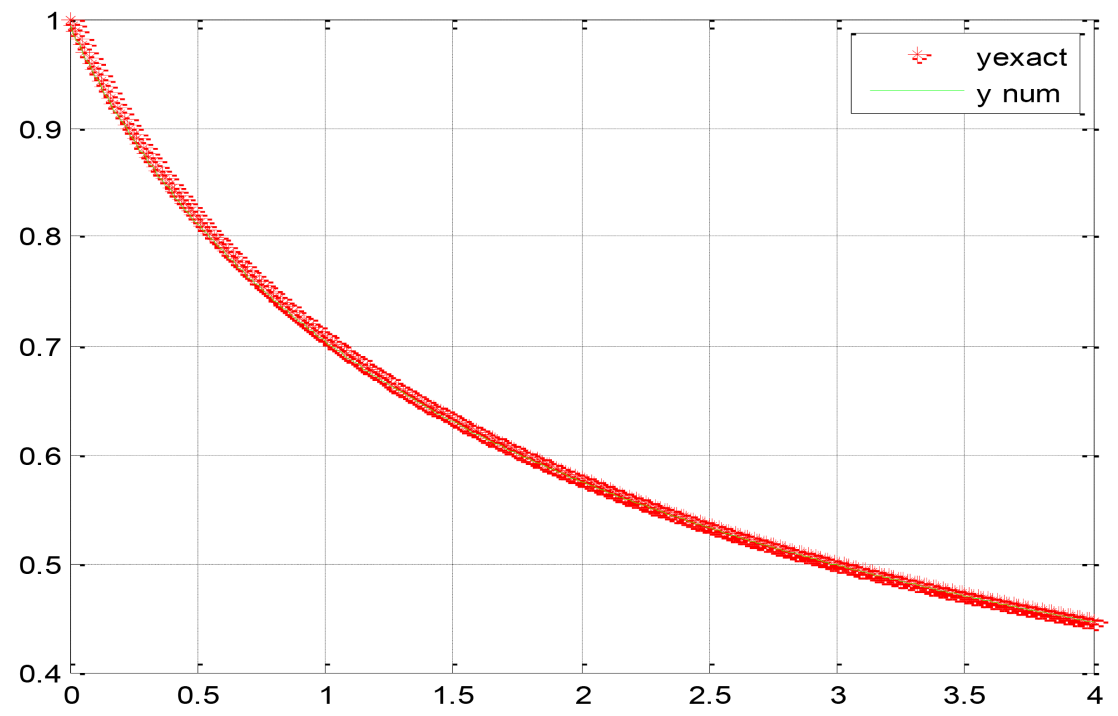

Figure 4. Numerical and exact solutions for problem 4. 


\section{Conclusion}

In this paper, SDBM for the solution of first order IVP in ODEs is proposed. The methods are found to be consistent and zero stable. Furthermore, the result obtained in Figure 2 shows that some of the SDBM proposed herein are A-stable and L-stable, while some are $A(\alpha)$-stable. Flowchart algorithm for the development of the SDBM is also presented herein. The SDBM is found to be suitable for the integration of stiff first-order IVP when applied on classical problems earlier studied in Zabidi et al. [30], Hairer et al. [13], Akinfenwa et al. [2].

\section{Acknowledgment}

The authors are grateful to Professor S. N. Jator of the Department of Mathematics and Statistics, Austin Peay State University, Clarksville, USA for the help in the implementation process.

\section{References}

[1] I. J. Ajie, P. Onumanyi and M. N. O. Ikhile, A family of one-block implicit multistep backward Euler type methods, America Journal of Applied and Computation Mathematics 4(2) (2014), 51-59.

[2] O. A. Akinfenwa, S. N. Jator and N. M. Yao, A self starting block Adams methods for solving stiff ordinary differential equation, The 14th IEEE International Conference on Computational Science and Engineering 26 (2011), 127-134.

[3] J. C. Butcher, Numerical Methods for Ordinary Differential Equations, Chichester: John Willey \& Sons, Ltd., 2008.

[4] J. R. Cash, Second derivative extended backward differential formulas for the numerical integration of stiff systems, SIAM J. Numer. Anal. 18 (1981), 21-36.

[5] P. Chartier, L-stable parallel one block methods for ordinary differential equations, SIAM J. Numer. Anal. 1(2) (1994), 552-571.

[6] C. F. Curtiss and J. O. Hirschfelder, Integration of stiff equation, Proc. Nat. Acad. Sci. 38 (1952), 235-243.

[7] G. Dahlquist, A special stability problem for linear multistep methods, BIT 3 (1963), 2743. 
[8] J. O. Ehigie and S. A. Okunuga, A stiffly stable second derivative block multistep formula with Chebyshev collocation points for stiff problems, International Journal of Pure and Applied Mathematics 4 (2014), 457-481.

[9] S. O. Fatunla, Numerical Methods for Initial Value problems in Ordinary Differential Equations, UK: Academic Press, Inc., 1988.

[10] W. H. Enright, Second derivative multistep method for stiff ordinary differential equations, SIAM J. Numer. Anal. 11 (1974), 321-331.

[11] C. W. Gear, Numerical Initial Value Problems in Ordinary Differential Equations, Englewood Cliffs, NJ: Prentice-Hall, 1971.

[12] G. K. Gupta, Implementing second derivative multistep methods using the Nordsieck polynomial representation, Math. Comp. 32 (1978), 13-18.

[13] E. Hairer, S. Norsett and G. Wanner, Solving Ordinary Differential Equations I: Stiff and Differential-Algebraic Problems 2, Germany: Springer-Verlag, 1991.

[14] E. Hairer and G. Wanner, Solving Ordinary Differential Equations II: Stiff and Differential-Algebraic Problems, 2nd ed., Germany: Springer Verlag, 1996.

[15] G. Hojjatti, M. Y. R. Ardabili, and S. M. Hosseini, New second derivative multistep methods for stiff system, Appl. Math. Model. 30 (2006), 466- 476.

[16] O. M. Ibrahim and M. N. O. Ikhile, On the construction of high accuracy symmetric super-implicit hybrid formulas with phase-lag properties, Transaction of Nigerian Association of Mathematical Physics 4 (2017), 101-108.

[17] O. M. Ibrahim and M. N. O. Ikhile, Highly stable super-implicit hybrid methods for special second order IVPs, American Journal of Applied Scientific Research 3(3) (2017), 21-27; doi: 10.11648/j.ajasr.20170303.11.

[18] G. Ismail, and I. Ibrahim, New efficient second derivative multistep methods for stiff systems, Appl. Math. Model. 23 (1999), 279-288.

[19] J. D. Lambert, Computational Methods for Ordinary Differential Systems, The Initial Value Problems, Chichester: Wiley, 1973.

[20] G. M. Kumleng, and U. W. Sirisena, $\mathrm{A}(\alpha)$ stable order ten second derivative block multistep method for stiff initial value problems, Int. J. Math. Stat. 10 (2014), 37-43.

[21] Z. Majid, M. Suleiman, F. Ismail and M. Othman, Two point block one-step method half Guass-Seidel for solving first-order ordinary differential equations, Mathematika 19 (2004), 91-100. 
[22] K. M. Mehdizadeh, N. N. Oskuyi and G. Hojjatti, A class of second derivative multistep method for stiff system, Acta Universitatis 30 (2012), 171-188.

[23] K. M. Mehdizadeh and M. Molayi, The class of A-stable hybrid multistep methods for numerical solution of stiff initial value problem, Mathematical Theory, and Modeling 5 (2015), 97-103.

[24] K. O. Muka and M. N. O. Ikhile, Second derivative parallel one block two points stabilized Simpson's method, Journal of Interdisciplinary Mathematics 13 (2010), 186191.

[25] N. A. M. Nasir, Z. B. Ibrahim, K. I. Othman and M. Suleiman, Solution of first-order stiff ordinary differential equations using fifth order block backward differentiation formulas, Sains Malaysiana 41 (2012), 489-492.

[26] F. F. Ngwane and S. N. Jator, Block hybrid second derivative method for stiff systems, International Journal of Pure and Applied Mathematics 80 (2012), 543-559.

[27] R. I. Okuonghae and M. N. O. Ikhile, A family of highly stable second derivative block methods for stiff IVPs in ODEs, Numer. Anal. Appl. 17 (2014), 67-81.

[28] J. B. Rosser, A Runge-Kutta for all seasons, SIAM Rev. 9 (1967), 417-452.

[29] R. K. Sahi, S. N. Jator and N. A. Khan, A Simpson's type second derivative method for stiff systems, International Journal of Pure and Applied Mathematics 81 (2012), 619633.

[30] M. Z. M. Zabidi, Z. A. Majid and N. Senu, Solving stiff differential equations using Astable block method, International Journal of Pure and Applied Mathematics 93(3) (2014), 409-425.

[31] H. Musa, M. B. Suleiman and N. Senu, Fully implicit 3-point block extended backward differentiation formula for stiff initial value problems, Appl. Math. Sci. 6(85) (2012), 4211-4228.

[32] J. M. Borwein and M. P. Skerritt, An Introduction to Modern Mathematical Computing with Mathematica, Springer, 2012.

[33] S. R. Otto and J. P. Denier, An Introduction to Programming and Numerical methods in MATLAB, Springer-Verlag, 2005. 